\title{
Continuous Class-F Power Amplifier Design by Using Multi-Harmonic Bilateral Pull
}

\author{
Chaoran Dong ${ }^{a}$, Yonglun Luo ${ }^{\mathrm{b}}$ and Qian Wang ${ }^{\mathrm{c}}$ \\ University of Electronic Science and Technology of China, Sichuan 611731, China \\ a604138041@qq.com, blyluo@uestc.edu.cn, c792637728@qq.com
}

Keywords: Continuous Class-F mode; multi-harmonic bilateral pull; high efficiency; broadband

\begin{abstract}
In this paper, multi-harmonic bilateral pull is used to design power amplifier(PA) fundamental and harmonics matching networks. By combining with continuous Class-F operation mode to realize high efficiency with broadband. And large signal dynamic device model is used in multi-harmonic bilateral pull to solve gain and efficiency decrease problem caused by the parasitic and nonlinear parameters. To verify the feasibility, CGH40010F device is used in the experimentation. The measured drain efficiency of the fabricated is between $64 \%$ and $75 \%$ from 1.5 to $2.5 \mathrm{GHz}(50 \%$ bandwidth) with the gain is $9.9-11.8 \mathrm{~dB}$.
\end{abstract}

\section{Introduction}

As the most important part of modern wireless communication system, RF PA directly impacts the performance of the wireless transceiver system. In the background of global advocacy of green environmental protection, energy saving and emission reduction is imperative. In addition, transmission rates, amounts of information and quality of signals are constantly increasing. These make broadband with high efficiency PA become an urgent need [1]. But it is difficult to make PA realize high efficiency with broadband. To solve this problem, V. Carrubba etc proposed continuous class-F PA that could achieve high efficiency with broadband based on waveform engineering in 2010 [2].

But controlling the harmonics at each frequency point in whole bandwidth is very difficult. At the same time, parasitic parameters and nonlinear parameters limits the performance of PA [3]. To solve these problems, multi-harmonic bilateral pull with large signal dynamic device model should be used in PA design.

\section{Theory}

\subsection{Continuous Class-F PA.}

The drain voltage expressions of continuous Class-F PA is (until third harmonic) [4]:

$$
\mathrm{V}_{\mathrm{ds}}(\theta)=\left(1-\frac{2}{\sqrt{3}} \cos \theta\right)^{2}\left(1-\frac{2}{\sqrt{3}} \cos \theta\right)(1-\gamma \sin \theta)
$$

The fundamental, second and third harmonic impedances are:

$$
\begin{aligned}
\mathrm{Z}_{1, \mathrm{i}} & =\left(\frac{2}{\sqrt{3}}+\mathrm{j} \gamma\right) \mathrm{R}_{\mathrm{opt}} \\
\mathrm{Z}_{2, \mathrm{i}} & =\left(-\mathrm{j} \frac{7 \pi \gamma}{8 \sqrt{3}}\right) \mathrm{R}_{\mathrm{opt}} \\
\mathrm{Z}_{3, \mathrm{i}} & =\infty
\end{aligned}
$$

The drain voltage formula of continuous class-F PA is the drain voltage formula of class-F PA multiplied by the expansion factor $(1-\gamma \sin \theta)$. The range of $\gamma$ must be -1 to 1 in order to ensure $\mathrm{V}_{\mathrm{ds}}, 0$. The idea of continuous PA is to replace a single matching point with high efficiency impedance space. No matter how $\gamma$ changes in its range, the real part of the fundamental, second and third harmonic impedances are invariable. As long as the output impedance is contained in the impedance space of the corresponding frequency point, drain voltage and current waveform of PA can be interlaced to achieve same efficiency as traditional class-F PA. And with the change of $\gamma$, bandwidth of PA is increased. The drain voltage waveform with different $\gamma$ value is shown in Fig. 1. 


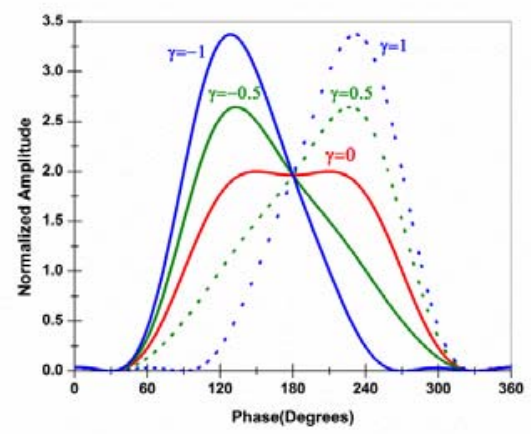

Fig.1 The drain voltage waveform with different $\gamma$

\subsection{Multi-Harmonic Bilateral Pull.}

Multi-harmonic bilateral pull can simulate the effects of the circuit performance under different harmonic impedances of each harmonics, and find out the best impedance of each harmonics[5]. The simulation formula is described as:

$$
\mathrm{E}(\mathrm{X}, \mathrm{Z})=0
$$

Among them, $\mathrm{E}$ is harmonic balance $(\mathrm{HB})$ error vector; $\mathrm{X}$ is all harmonics state variables; $\mathrm{Z}$ is the harmonics load of all external ports. Then the kth sub vector of $E$ is:

$$
\mathrm{E}_{\mathrm{k}}(\mathrm{X}, \mathrm{Z})=\mathrm{A}\left(\mathrm{k} \omega_{0}, \mathrm{Z}\right) \Phi(\mathrm{X}, \mathrm{Z})+\mathrm{B}\left(\mathrm{k} \omega_{0}, \mathrm{Z}\right) \Psi(\mathrm{X}, \mathrm{Z})+\mathrm{D}\left(\mathrm{k} \omega_{0}, \mathrm{Z}\right)
$$

Among them, $0, \mathrm{k}, \mathrm{N}$ ( $\mathrm{N}$ is the number of harmonics); $\mathrm{A}$ and $\mathrm{B}$ are circuit matrixes; $\mathrm{D}$ is inspirit function; $\Phi$ and $\Psi$ are the nonlinear sub-network ports harmonic vectors of instantaneous voltage and instantaneous current; $\mathrm{Z}$ is the harmonic load, the matrix is:

$$
\mathrm{Z}=\left|\mathrm{Z}_{\mathrm{L}}\left(0 \omega_{0}\right) \mathrm{Z}_{\mathrm{L}}\left(1 \omega_{0}\right) \cdots \mathrm{Z}_{\mathrm{L}}\left(\mathrm{k} \omega_{0}\right) \cdots \mathrm{Z}_{\mathrm{L}}\left[(\mathrm{n}-1) \omega_{0}\right] \mathrm{Z}_{\mathrm{L}}\left(\mathrm{n} \omega_{0}\right)\right|
$$

In the formula, $\mathrm{Z}_{\mathrm{L}}\left(\mathrm{k} \omega_{0}\right)$ is the $\mathrm{k}$ th harmonic load of the load port; $\mathrm{Z}_{\mathrm{L}}\left(0 \omega_{0}\right)$ represents the DC component. Through the solution of the Z-matrix, circuit performance can be obtained in different fundamental and harmonics impedance conditions.

\section{PA Design}

\subsection{High Efficiency Impedance Space Analysis.}

The fundamental and harmonics impedances of continuous class-F PA obtained by the formula (2) ignore parasitic and nonlinear parameters of device. This makes the impedance of continuous classF PA deviate from high efficiency impedance space in actual operation which also leads to the efficiency decrease.

Large signal dynamic device model contain parasitic and nonlinear parameters at each frequency point. To avoid the above problems, fundamental and harmonic high efficiency impedances space of the PA should obtained by multi-harmonic bilateral pull using CGH40010F large signal dynamic model. Fundamental load-pull is carried out with $200 \mathrm{MHz}$ step in $1.5-2.5 \mathrm{GHz}$. At this time, the loadpull result of fundamental impedance space is affected by input network. So source-pull should use the result of load-pull. Load-pull and source-pull should be iterated repeatedly until the result of loadpull and source-pull converge. Then using the result of fundamental impedance to do load-pull and source-pull for each harmonics at the same way.

Finally, the input and output high efficiency impedance region of fundamental and second harmonic can be obtained. The region which PAE, $70 \%$ of output fundamental and second harmonic as shown in Fig.2. Third harmonic impedance with each frequency point can get high efficiency in large region in 1.5-2.5GHz. So third and more harmonics should be ignored in PA design.The high efficiency regions of fundamental and second harmonic at each frequency point altogether consist of impedance space. 

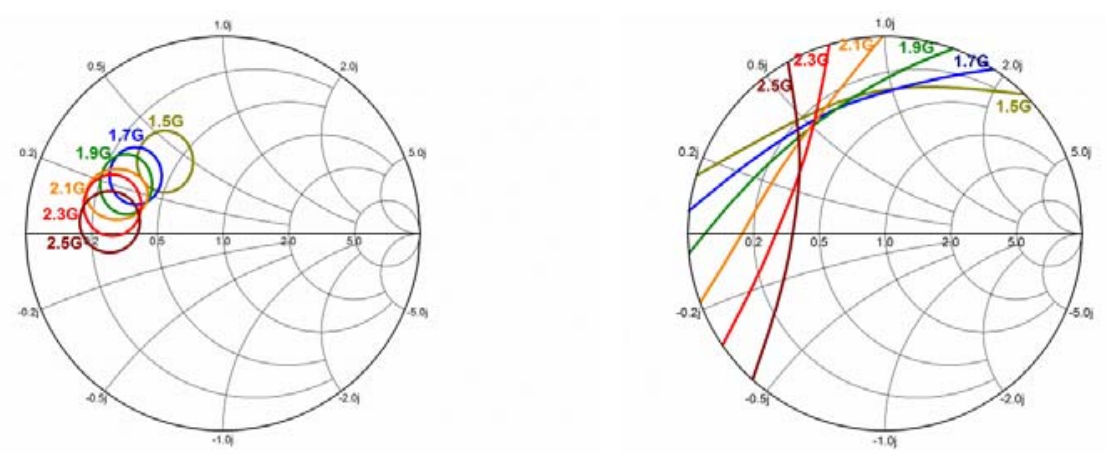

(a) output fundamental PAE, 70\% region (b) output second harmonic PAE,70\% region

Fig.2 high efficiency region of each frequent point

\subsection{Matching Network Design.}

To the design of broadband PA, designing input and output matching network is very important. Matching network directly affect performance of PA at each frequency point.

In this design of continuous class-F PA, high efficiency should be obtained in the broadband of $1.5-2.5 \mathrm{GHz}$. And the fundamental and second harmonic impedance at each frequent point should be matched in its high efficiency impedance region. Stability network composed of LC should be add first to ensure PA does not occur oscillation. Harmonic control network should add in output matching network to control second harmonic impedance in high efficiency impedance region in Fig2(a) at each frequent point. Then design matching network combined with the harmonic control network to let fundamental impedance in high efficiency impedance region in Fig2(b) and match to $50 \Omega$. At last, output matching network frequency response as shown in Figure 3. Input network also use the same way to match fundamental and second harmonic impedance.

After finishing input and output matching network design, gain and efficiency of PA need to optimize by tuning various parts of the microstrip line until the PA get high efficiency in its whole bandwidth.

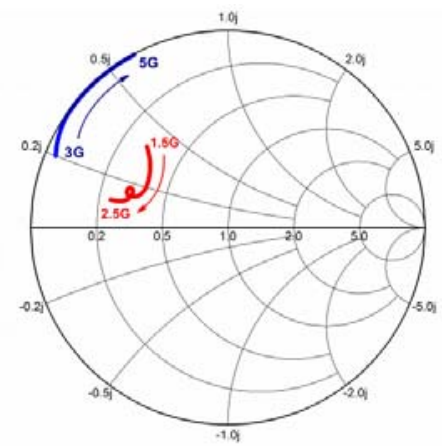

Fig.3 Output matching network frequency response

\section{PA Design}

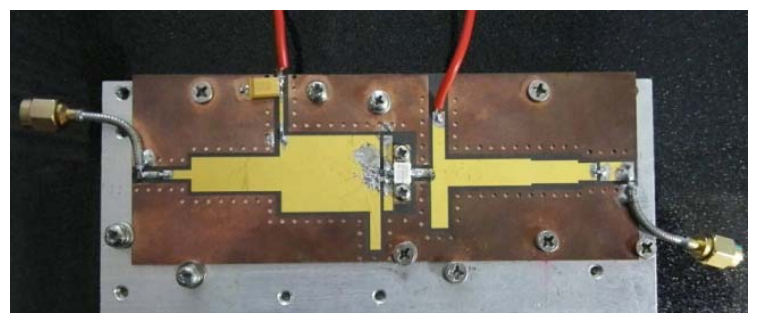

Fig.4 final fabricated PA

The board of PA is RO4350B which thickness of $0.508 \mathrm{~mm}$, $\varepsilon \mathrm{r}=3.66$, and gold thickness of $3 \mathrm{um}$. The final fabricated PA is shown in Fig.4. The CGH40010F packaged device was chosen for experimentation. The PA was biased at $-2.8 \mathrm{~V}$ on the gate with $28 \mathrm{~V}$ on the drain. Continuous wave (CW) excitation from $1.5-2.5 \mathrm{GHz}$ was chosen to test the PA. Fig.5 shows the measure result of drain efficiency and gain in actual test. It can be seen that the drain efficiency of this continuous class-F 
PA in $1.5-2.5 \mathrm{GHz}$ is $64 \%-75 \%$, and the gain is $9.9-11.8 \mathrm{~dB}$. Table 1 shows the performance comparison between this paper and other recent continuous class-F PA designed by [6,7].

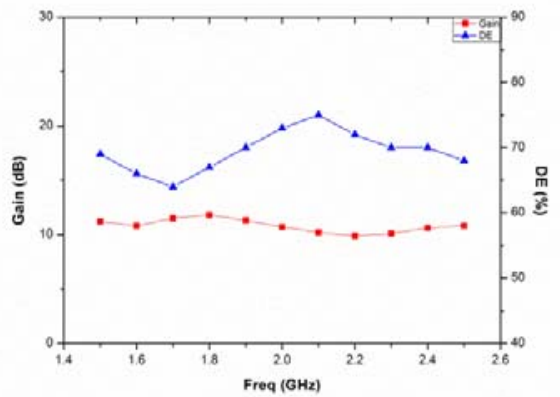

Fig.5 Measured drain efficiency and gain

Table 1 Comparison with other recent continuous Class-F PAs

\begin{tabular}{|ccccc|}
\hline Ref & Year & BW $(\mathrm{GHz})$ & $\mathrm{DE}(\%)$ & $\mathrm{Gain}(\mathrm{db})$ \\
\hline$[6]$ & 2016 & $1.4-2.4$ & $58-72$ & $9.5-10.5$ \\
{$[7]$} & 2017 & $1.5-2.5$ & $60-73.5$ & $9.5-12.7$ \\
This work & & $1.5-2.5$ & $64-75$ & $9.9-11.8$ \\
\hline
\end{tabular}

\section{Summary}

In this paper, a continuous Class-F PA was designed and implemented. Multi-harmonic bilateral pull was used to design fundamental and harmonics matching network to realize high efficiency. And large signal dynamic device model is used in multi-harmonic bilateral pull to solve problem of gain and efficiency decrease caused by the parasitic and nonlinear parameters. The experimental result show that high efficiency with broadband PA was realized.

\section{References}

[1]. S. C. Cripps. RF power amplifiers for wireless communications. Artech House Inc., 2006, p7-8.

[2]. V. Carrubba, A. L. Clarke, M. Akmal, et al. The continuous class-F mode power amplifier. IEEE Microwave Conference. European, 2010, p. 1674-1677

[3]. V. Carrubba, M. Akmal, P. J. Tasker, et al. On the extension of the continuous class-f mode power amplifier. Microwave Theory and Technology. Vol.59 (2011), No. 5, p. 1294-1303.

[4]. S. C. Cripps, P. J. Tasker, A. L. Clarke, et al. On the continuity of high efficiency modes in linear RF power amplifiers. Microwave and Wireless Components Letters. Vol.19 (2009), No. 10, p.665-667.

[5]. H. Chen and Y. X. Zhang. Design of Microwave Power Amplifier Based on Multi-Harmonic Bilateral-Pull Technology. Journal of Microwaves, Vol.24 (2008), No. 3, p.57-60.

[6]. E. Aggrawal, S. Saxena and K. Rawat. Broadband Power Amplifier Design by Exploring Design Space of Continuous Class-F mode. Proceedings of the Asia-Pacific Microwave Conference. Roorkee, 2016, p.1-4.

[7]. E. Aggrawal, K. Rawat and P. Roblin. Investigating Continuous Class-F Power Amplifier Using Nonlinear Embedding Model. Microwave and wireless components letters, Vol. 27 (2017), No. 6, p.593-595. 